1 Title: Abiotic conditions shape spatial and temporal morphological variation

\title{
in North American birds
}

3 Authors: Casey Youngflesh ${ }^{1, \dagger}$, James F. Saracco ${ }^{2}$, Rodney B. Siegel ${ }^{2}$, Morgan W. Tingley ${ }^{1}$

4

5 Affiliations:

$6{ }^{1}$ Department of Ecology and Evolutionary Biology, University of California, Los Angeles, CA

7 90095, USA

$8 \quad{ }^{2}$ The Institute for Bird Populations, Petaluma, CA 94953, USA

$9 \quad{ }^{\dagger}$ Corresponding author: cyoungflesh@ucla.edu

10

11 Classification:

12 Biological Sciences: Ecology

13

14 Keywords:

15 Bergmann's Rule, morphology, intraspecific variation, global change, hierarchical Bayesian

16 modeling 


\section{Abstract:}

18 Abiotic environmental conditions play a key role in driving the size and shape of organisms.

19 Quantifying environment-morphology relationships is important not only for understanding the

20 fundamental processes driving phenotypic diversity within and among species (1), but also for

21 predicting how species will respond to ongoing global change (2). Despite a clear set of

22 expectations motivated by ecological theory (3), broad evidence in support of generalizable

23 effects of abiotic conditions, such as temperature (4), on spatial and temporal intraspecific

24 morphological variation has been limited. Using standardized data from over 250,000 captures of

25105 landbird species, we assessed intraspecific shifts in bird morphology since 1989 while

26 simultaneously measuring spatial morphological gradients across the North American continent.

27 Across bird species, we found strong spatial and temporal trends in body size, with warmer

28 temperatures associated with smaller body sizes both at more equatorial latitudes and in more

29 recent years. The magnitude of these thermal effects varied both across and within species, with

30 results suggesting it is the warmest, rather than the coldest, temperatures driving both spatial and

31 temporal trends. Across elevation, we found that body size declines as relative wing length

32 increases, likely due to the benefits that longer wings confer for flight in thin air environments.

33 Our results provide support for both existing and new large-scale ecomorphological gradients

34 and highlight how the response of functional tradeoffs to abiotic variation drives morphological

35 change.

\section{Significance Statement:}

38 Characterizing how the size and shape of organisms varies over space and time is key to

39 understanding the processes that create ecological communities and for predicting how species 
40 will respond to climate change. Across more than 100 species of North American birds, we show

41 that within species the size and shape of individuals varies substantially across space and time.

42 Warmer temperatures are associated with smaller body sizes, likely due to the importance of

43 body size for thermoregulation. As the climate continues to warm, these species will likely

44 continue to shrink. We also provide the first large-scale evidence of an increase in wing length

45 with elevation, a pattern that could be attributed to thinner air in high elevation environments.

47 Main text:

\section{INTRODUCTION}

49 Morphology is both a cause (5) and a consequence (6) of how organisms interact with their

50 environment. Assessing patterns in morphological variation both across and within (7) species

51 provides a means to better understand these interactions, and consequently, predict ecological

52 responses to environmental change. Ecological theory suggests that both the sizes and shapes of

53 organisms should vary across latitude [e.g., Bergmann's (3) and Allen's (8) Rules] and also

54 possibly elevation, particularly for flying organisms [due to lower temperatures and lower air

55 density at high elevations (9)]. These ecogeographic expectations are commonly used to

56 motivate hypotheses for how species will respond to climate change (10), such as the suggestion

57 that declining body size may be a generalized response of endotherms to warming temperatures

58 (2). However, broad evidence in support of generalizable effects of abiotic conditions on

59 intraspecific spatial and temporal morphological variation has been limited by a lack of

60 taxonomic and spatial replication $(2,11-13)$, yielding conflicting results. For birds in particular,

61 which have precipitously declined in North America over a period coincident with modern 
62 anthropogenic warming (14), much remains unknown regarding how abiotic factors may shape

63 morphological traits over space and time.

64 We evaluated spatiotemporal morphological variation in 105 North American bird

65 species over 30 years (1989-2018), across more than 43 degrees of latitude and nearly 3,000 m

66 of elevation, using data from more than 250,000 live birds, primarily passerines or near-

67 passerines, captured during the breeding season using standardized methods (15) (Fig. 1A, S1,

68 Table S1). These measurements comprise a dataset on bird morphology that is unparalleled in

69 size, taxonomic diversity, and spatiotemporal scope. Combining field measures of body mass

70 and wing length (i.e., length of the unflattened, closed wing) with allometric scaling theory (16),

71 we derived two morphological indices, a Size Index (SI) and a Wing Index (WI)(Fig. 1B, 1C).

72 SI and WI reflect overall bird body size and "wingyness" (wing length relative to body mass),

73 respectively (Fig. S2), and were used to account for the fact that mass and wing length are

74 intrinsically linked (i.e., that changes in mass may be due to changes in wing length and vice

75 versa). Using a hierarchical Bayesian approach to estimate species-specific responses, we

76 modeled these indices as a function of year, latitude, and elevation, and estimated the impact of

77 spatial and temporal variation in temperature on bird body size.

\section{RESULTS}

80 Across the wide spatial and taxonomic breadth of sampling, avian body size decreased over time

$81\left(S I \mu_{\eta_{I D X}}[\mathrm{Eq} \mathrm{10]}=-0.03\right.$ SI per 10 years, 89\% CI: $[-0.04,-0.01], p(<0)=1$; Fig. 2A, S3A,

82 Table S2). Absolute body mass showed range-wide declines of up to $2.78 \%$ between 1989 and

832018 (e.g., tree swallow Tachycineta bicolor, $\omega_{M_{T I M E}}[\mathrm{Eq} 25]=-2.78,89 \%$ CI: [-4.98, -0.63],

$84 p(<0)=0.98)$, with a mean decline in mass of $0.56 \%$ across all 105 species $\left(\mu_{\omega_{M}{ }_{T M E}}[\mathrm{Eq} 26]=\right.$ 
$85-0.56,89 \%$ CI: $[-0.78,-0.34], p(<0)=1$; Fig. S7A, Table S3). This temporal trend toward

86 smaller bodies, replicated across species and over most of a continent, is likely the result of

87 warming summer temperatures. Specifically, smaller body sizes were associated with elevated

88 June maximum temperatures in the year of capture $\left(\mu_{\gamma_{T V T}}\right.$ lag-0 [Eq 15] $=-0.012$ SI per $1^{\circ} \mathrm{C}$,

$8989 \%$ CI: $[-0.014,-0.009], p(<0)=1)$, as well as one year prior to capture $\left(\mu_{\gamma_{T V T}}\right.$ lag-1 $=$ -

$900.004,89 \% \mathrm{CI}:[-0.007,-0.001], p(<0)=0.99)$. Temperatures two years prior to capture were

91 not strongly related to body size $\left(\mu_{\gamma_{T V T}}\right.$ lag-2 $=-0.001,89 \%$ CI: $[-0.003,0.001], p(<0)=0.73$;

92 Fig. 2B, S5, Table S4). Temperatures one and two years prior to capture correspond to

93 environmental conditions likely experienced during ontogenesis, although post-natal dispersal

94 limits the strength of this inference from banding data. However, these findings align with

95 expectations, given that smaller-bodied individuals - having larger surface-area-to-volume ratios

96 - tend to have lower cooling costs compared to larger-bodied individuals, and provide strong

97 support for the hypothesis that shrinking body size is a generalized response to climate change

$98(2,10)$.

99 Temperature-mediated size-dependent mortality [which may result in directional

100 selection, conditional on the heritability of body size; e.g., (17)] and/or developmental plasticity

101 during early life stages (18) may be the most likely proximate drivers of our finding of an

102 association between warmer summers and smaller bodies. While widespread evidence for

103 adaptive evolutionary responses to climate change is somewhat limited (19), the rate of

104 morphological change reported here is within the range that might be expected via evolutionary

105 change (Fig. S10). The fact that conditions in the year of capture have the strongest effect on

106 body size may indicate that temperature impacts morphology most strongly via size-dependent

107 mortality of adult birds. The lack of a strong relationship with temperatures two years prior to 
capture could suggest that a large portion of measured individuals were in their second year of life and never experienced the conditions 24 months prior. Greater effects of temperature on

110 body size in the warmer portions of species' ranges $\left(\mu_{\theta_{T V T}}\right.$ lag-0 [Eq 15] $=-0.011$ unit change in

111 effect of temperature per $10^{\circ} \mathrm{C}$ change in mean site temperature, $89 \% \mathrm{CI}$ : $[-0.020,-0.002], p(<$

$1120)=0.97$; Fig. 2C) suggests that it is the hottest experienced temperatures - rather than the

113 coldest - driving this body size-temperature association (20). This effect was less pronounced for

114 temperatures in the year prior and two years prior to capture $\left(\mu_{\theta_{T V T}}\right.$ lag- $1=-0.007,[89 \% \mathrm{CI}$ : -

$0.017,0.002], p(<0)=0.90 ; \mu_{\theta_{T V T}}$ lag-2 $=-0.004,89 \% \mathrm{CI}:[-0.014,0.005], p(<0)=0.75 ;$ Fig.

2C). While poleward range shifts of species could also result in directional change in

117 morphology at a given location, declines in body size in even the warmest portions of species'

118 ranges (where individuals are generally smallest) suggests that dispersal is not the primary

119 mechanism driving these observed changes.

121 (wing length relative to body mass) of birds has increased over time $\left(W I \mu_{\eta_{I D X}}[\mathrm{Eq} \mathrm{10]}=\right.$

1220.02 WI per 10 years, 89\% CI: [0.00, 0.03], $p(>0)=0.95$; Fig. S4A, Table S2). While this

123 pattern could be due to changing migratory patterns in response to ongoing range shifts (21),

124 constraints on the rate at which wing length can change over time compared to body size $(22,23)$

125 might also play a role. Specifically, in contrast to previous findings that relied on bird specimens

126 derived from a single migratory bottleneck (13), we observed no change in absolute wing length

127 over time - temporal changes in wingyness were the result of declining mass $\left(\mu_{\omega_{M}{ }_{T I M E}}[\mathrm{Eq} 26]=\right.$

$128-0.56 \%$ change over study period, $89 \% \mathrm{CI}:[-0.78,-0.34], p(<0)=1)$ with relatively stable wing

129 length $\left(\mu_{\omega_{W_{T I M E}}}[\mathrm{Eq} 26]=-0.01 \%\right.$ change over study period, $89 \% \mathrm{CI}:[-0.13,0.12], p(<0)=$ 
0.54; Fig. S7A, S8A, Table S3). That is, while birds have overall gotten smaller, their wings have stayed relatively the same size.

Why is it so critical to control for geography when assessing temporal trends in

133 phenotypes? Bird morphology shows strong and generalizable trends in morphology over both

134 latitude and elevation. As illustrated by our dataset across 105 bird species and most of a

135 continent, body size strongly increases with latitude $\left(S I \mu_{\gamma_{I D X}}[\mathrm{Eq} 11]=0.37\right.$ SI per $10^{\circ}$ latitude,

$13689 \%$ CI: $[0.29,0.45], p(>0)=1$; Fig. 3A, S3B, Table S2), supporting the intraspecific

137 interpretation of Bergmann's Rule (3), despite decades of debate on its relevance (4). Across

138 species' latitudinal ranges, body mass increases 5.72\% $\left(\mu_{\omega_{M_{L A T}}}[\mathrm{Eq} 26]=5.72 \%, 89 \% \mathrm{CI}\right.$ : [5.39,

139 6.04], $p>0)=1$; Fig. S7B, Table S3). Larger body sizes are associated with regions with cooler

140 average temperatures $\left(S I \mu_{\beta_{S V T}}[\mathrm{Eq} 20]=-0.36 S I\right.$ per $10^{\circ} \mathrm{C}$ change in mean site temperature,

$14189 \%$ CI: $[-0.44,-0.27], p(<0)=1$; Fig. 3B, S6) that are generally found at higher latitudes,

142 supporting the notion that thermal factors play a substantial role in governing body size not only

143 over time, but also over space (20). Additionally, we found that this relationship between

144 temperature and spatial variation in body size is stronger for species that experience warmer

145 conditions $\left(\theta_{S R}\right.$ [Eq 22] $=-0.30$ unit change in effect of temperature per $10^{\circ} \mathrm{C}$ change in mean

146 range-wide temperature, 89\% CI: $[-0.49,-0.10], p(<0)=0.99$; Fig. 3B), illustrating - as with

147 findings of temporal associations between body size and temperature - that the warmest, rather

148 than the coldest, temperatures likely drive intraspecific adherence to Bergmann's Rule.

Factors other than temperature may also be important in driving morphological variation.

150 For example, some evidence exists for an increase in wingyness with latitude (WI $\mu_{\gamma_{I D X}}[\mathrm{Eq} 11]$

$151=0.04 \mathrm{WI}$ per $10^{\circ}$ latitude, $89 \% \mathrm{CI}$ : [0.00, 0.07], $p(>0)=0.92$; Fig. S4B, Table S2). While

152 thermal factors might suggest that appendages should be smaller towards the poles to limit heat 
153 loss - known as Allen's rule (8) - bird wings are not thermoregulatory organs. The length of the

154 closed bird wing is primarily a function of flight feather length. Relatively longer wings at higher

155 latitudes may reflect the longer distances that breeding birds from more northerly populations

156 tend to travel to complete their migration. Longer and more pointed wings are thought to

157 increase the efficiency of long flights and are generally found in populations that migrate longer

158 distances (24). For some species, populations breeding at higher latitudes migrate farther than

159 southern populations, yielding 'leapfrog' migration patterns; for other species, equatorward

160 populations of an otherwise migratory species remain non-migratory (25). Indeed, species known

161 to exhibit leapfrog migrations [e.g., Wilson's warbler Cardellina pusilla: (26), fox sparrow

162 Passerella iliaca: (27)], as well as migratory species with resident populations in the southern

163 portions of their ranges [e.g., Eastern towhee Pipilo erythrophthalmus: (28), white-eyed vireo

164 Vireo griseus: (28)], here show pronounced increases in wingyness with latitude (Fig. S4B,

165 Table S2). Smaller or even negative effects of latitude for other species might be indicative of

166 alternative migration strategies - in which northerly populations do not migrate longer distances

167 than southerly populations (25) - as well as the importance of other factors, such as variation in

168 habitat structure (29) and/or predation (30), that might also drive variation in wing length.

170 temperatures at high elevations, body size might be expected to increase (i.e., Bergmann's Rule

171 applied to elevation). However, we find that body size generally decreases with elevation (SI

$172 \mu_{\theta_{I D X}}[$ Eq 11] $=-0.06$ SI per 1000m, 89\% CI: $[-0.12,0.00], p(<0)=0.96$; Fig. S3C, Table S2),

173 indicating that, contrary to the general associations found between body size and temperature

174 over space, pressures unrelated to thermoregulation dominate over this gradient [potentially

175 reflecting lower resource availability at higher elevations (31)]. Species with wide elevational 
176 gradients may therefore rely on a variety of behavioral adaptations, including altitudinal

177 migration (32) and even nightly torpor (33), to cope with lower temperatures at higher

178 elevations.

179 In contrast to body size, wingyness strongly increases with elevation $\left(W I \mu_{\theta_{I D X}}[\mathrm{Eq} 11]=\right.$ in both indices are due to countervailing changes in absolute morphology: body mass decreases $\left(\mu_{\omega_{\text {ELEV }}}[\mathrm{Eq}\right.$ $[$ Eq 26] $=-1.15 \%$ change over species' elevational range, $89 \% \mathrm{CI}:[-1.42,-0.89]$, $p(<0)=1$; Fig. S7C, Table S3) while wing length increases with elevation $\left(\mu_{\omega_{W_{E L E V}}}[\mathrm{Eq} 26]=\right.$ ecogeographic relationships for birds are likely due to the key role that air pressure plays in flight performance. Air density, a key determinant in the amount of lift that a wing produces, is lower at higher elevations, necessitating some compensatory measures to maintain flight [i.e., more relative power output via larger wings and/or lower mass, larger wing stroke amplitude, or increased wingbeat frequency $(9,34)]$. individuals (35). Our results illustrate a clear increase in wing length with elevation independent

193 of any changes in body size (Fig. 4), providing large-scale, cross-taxonomic evidence for a new

194 ecomorphological gradient. This intraspecific pattern of increased wing length with elevation 195 harmonizes observations in some insects (36), among specific groups of bird species, including 196 hummingbirds [family Trochilidae (37)] and white-eyes [Zosterops spp. (34)], and from a limited 197 number of single-species studies [e.g., song sparrow Melospiza melodia (38), Eurasian tree 198 sparrow Passer montanus (39)]. 


\section{DISCUSSION}

201 While intraspecific morphological differences are often disregarded in macroecological and

202 functional studies [e.g., (40)], this important element of biodiversity has important implications

203 for understanding how organisms are shaped by their environments, how they are likely to

204 respond to future global change, and for the conservation of natural systems (41). For example,

205 the degree to which species can respond to the thermoregulatory pressures caused by warming

206 temperatures may impact their ability to persist in their current ranges (42). More frequent

207 extreme weather events that may result in large-scale thermoregulatory-related mortality events

208 (43) and chronic sub-lethal effects of increased temperature may have pronounced effects on

209 populations (44). While body size in North American birds has responded to warming

210 temperatures over time, larger responses to temperature variation over space compared to

211 temperature variation over time (change in $S I$ per $1^{\circ} \mathrm{C}$ was more than three times as large over

212 space compared to temperatures over time at Lag 0) suggests that the rate of morphological

213 change over time may be evolutionarily and/or plastically constrained - species may not be

214 responding rapidly enough over time to keep pace with ongoing climatic change (45). The

215 potential for mismatch between species and their environments is especially concerning for some

216 bird species - such as those found in desert environments - that may lack suitable microrefugia

217 to buffer them from especially warm temperatures (46).

218 Morphological responses to thermoregulatory pressures, as well as the importance of

219 flight efficiency, illustrate how interacting functional tradeoffs contribute to observed

220 morphological variation. Other factors not directly considered in this study, including conditions

221 experienced on overwintering grounds, likely act in concert with these processes. Characterizing 
222 the interplay between these various factors, operating over space and time, is key to

223 understanding how morphology is likely to change into the future, in response to continued

224 abiotic environmental change. Although the ecological consequences of morphological change

225 and how morphology interacts with other climate change responses - including shifts in species’

226 ranges (47) and the timing of seasonal events (48) - are currently unknown (2), the importance

227 of body size for life history traits (49), physiology (50), and both cross- (51) and intra-trophic

228 (52) interactions, suggests the implications of these changes could be far reaching. Given

229 projected changes in climatic conditions, continued morphological change and its associated

230 consequences can be expected into the foreseeable future.

232 MATERIALS AND METHODS

233 Morphological data

234 Bird morphology data were collected as part of the Monitoring Avian Productivity and

235 Survivorship (MAPS) program, a collaborative long-term bird-banding project operating across

236 North America (15). Data were obtained from 1124 banding stations (Fig. 1), each consisting of

237 6-20 mist nets, over the period 1989-2018 (though most stations operated during only a subset

238 of this period). Banding stations were operated 6-12 times per year, from May 1 to August 28

239 (15), encompassing the breeding season for most birds in North America. Only records obtained

240 within species' breeding ranges were used (as determined annually by banding station operators).

241 For each captured bird, wing length (distance between the carpal joint and the wing tip,

242 commonly referred to as unflattened wing chord) was measured to the nearest millimeter

243 following (53) and body mass was recorded to the nearest 0.5 grams (15). Birds were aged

244 following criteria summarized by (53). 
We restricted our analyses to male birds classified as After Hatch Year (captured at least

246 one breeding season after the hatch year of the bird) to avoid any confounding morphological

247 variation among age classes and between sexes and changes in female bird mass throughout the

248 season that may be due to egg production and laying. All records with body mass or wing length

249 measurements that were more than five median absolute deviations [MAD (54)] away from the

250 median were excluded, as these likely represented measurement or data entry errors. If an

251 individual was captured more than once in a season, only measurements taken during the initial

252 capture were considered. Only species for which data were available for at least 375 captures

253 (post data filtering) were analyzed. In total, morphological data from 253,488 captures of 105

254 species, representing two orders and 18 taxonomic families were used from banding stations

255 spanning more than 43 degrees of latitude $\left(26.1^{\circ} \mathrm{N}-69.4^{\circ} \mathrm{N}\right)$ and 2996 meters of elevation

256 (Table S1).

257

258 Elevation Data

259 Elevation data for each banding station were obtained from the 30 -arcsecond resolution

260 (approximately 1-km at the equator) Global Multi-resolution Terrain Elevation Data 2010 (55)

261 data product.

262

\section{Temperature Data}

264 Daily maximum temperature data for each banding station were obtained over the study period

265 from the 1-km gridded Daymet surface weather data product (56). For each year at each site, we

266 calculated the average maximum temperature from ordinal day (day of year) 152 to ordinal day

267181 (June 1 to June 30 in a non-leap year). We refer to this annual metric as 'June maximum 
temperature'. We calculated the mean June maximum temperature across years at each station as well as year-specific values for temperature at each station to evaluate the effect of temperature on morphological variation across space and time, respectively. Species-wide mean temperature

271 values were calculated by taking the mean June maximum temperature across all stations for 272 each species.

275 Two morphological indices were derived from data collected on body mass and wing length for 276 each bird. The Size Index (SI) corresponds to the overall size of an individual, while the Wing

277 Index (WI) corresponds to the relative (to body mass) wing length, or "wingyness", of each 278 individual. These indices were derived using the expected power law $(57,58)$ relationship

279 between these two traits,

$$
W=b M^{c},[1]
$$

281 where $W$ is wing length, $M$ is body mass, $b$ is a scalar, and $c$ is the scaling exponent (Fig. 1B, 282 1C, S2), denoting how rapidly wing length increases as a function of mass. This relationship is 283 linearized when taking the log of both sides of the equation,

$$
\log (W)=\log (b)+c \times \log (M) \cdot[2]
$$

285 Using species-level mean values for both $\log (W)$ and $\log (M)$, we estimated the scaling exponent

286 by applying a phylogenetic regression [to control for the effect of phylogenetic relatedness on

287 parameter estimates (59)] using the 'caper' package (60) in $\mathrm{R}(61)$ to the linearized form of the

288 power law relationship (Eq. 2). Species-level mean values were used because we were interested

289 in understanding the general relationship between wing length and body mass. This represents

290 the null expectation for how wing length covaries with mass. We estimated the scaling exponent 
291 for each of 100 phylogenetic trees for the species of interest obtained from BirdTree [(62)

292 www.birdtree.org] to account for uncertainty in the phylogenetic relatedness of these species.

293 The mean of the 100 estimates $($ mean $=0.333$, standard deviation $=0.002)$ of the empirical

294 relationship between wing length and body mass (i.e., the scaling exponent) was nearly identical

295 to the theoretical expectation, given isometric scaling principles (where $c=\frac{1}{3}$; mass is expected

296 to be proportional to volume, which scales as the cube of a linear dimension, such as wing

297 length), and similar to estimates from other studies $(63,64)$ (Fig. S2).

298 For each species, measurements of body mass and wing length of individual bird captures

299 were then reprojected onto new axes using a rotation matrix derived from the estimated scaling

300 exponent (i.e., the rate at which wing length is expected to change with body mass). The rotation

301 matrix was specified as,

302

$$
R=\left[\begin{array}{cc}
\cos (\theta) & -\sin (\theta) \\
\sin (\theta) & \cos (\theta)
\end{array}\right],[3]
$$

303 where $\theta$ is the amount (in radians) the data are to be rotated. We specified $\theta$ as the negative arc304 tangent of $c$ (as applying the arc-tangent function to the tangent of a triangle [the tangent being 305 equivalent to the slope of a line] produces the angle in radians). For each species, we applied the 306 rotation matrix to logged body mass $(L M)$ and logged wing length (LW), to reproject the data 307 onto new axes (Fig. S2),

$$
\left[\begin{array}{l}
x^{\prime} \\
y^{\prime}
\end{array}\right]=R\left[\begin{array}{l}
L M \\
L W
\end{array}\right] \cdot[4]
$$

309 These reprojected data $\left(x^{\prime}\right.$ and $\left.y^{\prime}\right)$ were standardized within species (i.e., centered and divided by 310 the standard deviation) to create two relative indices $(S I=$ size index; $W I=$ wing index $)$ that 311 represent the overall size of the individual, and the degree to which wing length deviates from its 312 expected value given the body mass of the individual, respectively, 


$$
\frac{x_{i k}^{\prime}-\overline{x_{k}^{\prime}}}{\sigma_{x^{\prime} k}}=S I_{i k}[5]
$$

$$
\frac{y_{i k}^{\prime}-\overline{y_{k}^{\prime}}}{\sigma_{y^{\prime} k}}=W I_{i k},
$$

315 where $\overline{x^{\prime}}$ and $\overline{y^{\prime}}$ represent the mean and $\sigma_{x}$, and $\sigma_{y^{\prime}}$ represent the standard deviation of $x^{\prime}$ and $y^{\prime}$,

316 respectively, for each species, $k$, and $i$ represents each bird capture. This approach allowed us to

317 account for the expected non-linear relationship among these traits when assessing

318 spatiotemporal change and provides a means by which to assess morphological deviations from

319 an expectation derived from empirical estimates rooted in scaling theory (16). SI values were

320 closely correlated with logged mass (mean correlation coefficient across species $=0.99$, range $=$

$3210.98-1) . W I$ values showed a strong correlation to logged wing length (mean correlation

322 coefficient across species $=0.75$, range $=0.49-0.88)$, though not as strong as the relationship

323 between $S I$ and logged mass.

326 We used a hierarchical Bayesian approach to determine how SI and WI varied within species as

327 a function of time, latitude, and elevation. We fit separate models for each index, that were

328 identical in structure. In each case, the index $\left(y_{I D X}\right)$ for capture $i$, at banding station $j$, for species

$329 k$ was modeled as t-distributed, as a linear function of time,

$$
y_{I D X_{i j k}} \sim t\left(v_{I D X}, \mu_{I D K_{i j k}}, \sigma_{I D X_{k}}\right),[6]
$$

$$
\mu_{I D X_{i j k}}=\alpha_{I D X_{k}}+\beta_{I D X_{j k}} \times y_{e a r_{i j k}}+\xi_{I D X_{j k}}
$$

332 where $\alpha_{I D X}$ is the species-level intercept term, $\beta_{I D X}$ is the effect of year on the response

333 variable, $\xi_{I D X}$ is the species-station intercept term, $\sigma_{I D X}$ is the species-specific process error, $v_{I D X}$

334 represents the degrees of freedom, controlling the normality of the distribution (resulting in a 
335 Cauchy distribution when $v_{I D X}=1$ and approaching a normal distribution as $v_{I D X}$ approaches

336 infinity), and the $I D X$ subscript denotes the association of that parameter with this model (to help

337 distinguish these parameters from those in other models). The degrees of freedom parameter of

338 the t-distribution allows for additional flexibility (compared with the normal distribution) in

339 modeling the structure of the residuals [for instance when there are 'extreme observations' (65)].

340 Parameter $\alpha_{I D X}$ was modeled as normally distributed,

$$
\alpha_{I D X_{k}} \sim N\left(\mu_{\alpha_{I D X}}, \sigma_{\alpha_{I D X}}\right),[7]
$$

342 where $\mu_{\alpha_{I D X}}$ and $\sigma_{\alpha_{I D X}}$ represent the mean and standard deviation of $\alpha_{I D X}$ across all species,

343 respectively. Parameter $\beta_{I D X}$ was modeled as normally distributed,

$$
\beta_{I D X_{j k}} \sim N\left(\eta_{I D X_{k}}, \sigma_{\beta_{I D X}}\right),[8]
$$

345 where $\eta_{I D X}$ represents the mean effect of year on the response for each species, and $\sigma_{\beta_{I D X}}$

346 represents the process error. Parameter $\sigma_{I D X}$ was modeled as half-normal (normal but with

347 support only over positive values),

$$
\sigma_{I D X_{k}} \sim H N\left(\tau_{\sigma_{I D X}}, \kappa_{\sigma_{I D X}}\right),[9]
$$

349 where $\tau_{\sigma_{I D X}}$ and $\kappa_{\sigma_{I D X}}$ represent the mean and standard deviation of $\sigma_{I D X}$, respectively. Process

350 error was modeled hierarchically, as the degree to which these explanatory variables explain

351 variation in the data may vary by species. Parameter $\eta_{I D X}$ was modeled as normally distributed,

$$
\eta_{I D X_{k}} \sim N\left(\mu_{\eta_{I D X}}, \sigma_{\eta_{I D X}}\right),[10]
$$

353 where $\mu_{\eta_{I D X}}$ and $\sigma_{\eta_{I D X}}$ represent the mean and standard deviation of $\eta_{I D X}$ across all species,

354 respectively. The species-station intercept term, $\xi_{I D X}$, was modeled as a linear function of

355 latitude and elevation,

$$
\xi_{I D X j k} \sim N\left(\mu_{\xi_{I D X}{ }_{j k}}, \sigma_{\xi_{I D X_{k}}}\right)[11]
$$




$$
\begin{gathered}
\mu_{\xi_{I D X} j k}=\gamma_{I D X_{k}} \times \operatorname{lat}_{j k}+\theta_{I D X_{k}} \times \text { elev }_{j k} \\
{\left[\begin{array}{l}
\gamma_{I D X_{k}} \\
\theta_{I D X_{k}}
\end{array}\right] \sim \operatorname{MVN}\left(\left[\begin{array}{c}
\mu_{\gamma_{I D X}} \\
\mu_{\theta_{I D X}}
\end{array}\right], \Sigma_{I D X}\right),}
\end{gathered}
$$

where $\gamma_{I D X}$ is the species-specific effect of latitude (lat) on $\xi_{I D X}, \theta_{I D X}$ is the species-specific effect of elevation (elev) on $\xi_{I D X}$, and $\sigma_{\xi_{I D X}}$ is the species-specific process error. Parameters

$361 \gamma_{I D X}$ and $\theta_{I D X}$ were modeled as multivariate normal, with means $\mu_{\gamma_{I D X}}$ and $\mu_{\theta_{I D X}}$, respectively, 362 and covariance $\Sigma_{\text {IDX }}$ (a 2 x 2 covariance matrix). Parameter $\sigma_{\xi_{I D X}}$ was modeled as half-normal

$$
\sigma_{\xi_{I D X}} \sim H N\left(\tau_{\sigma_{\xi_{I D X}}}, \kappa_{\sigma_{\xi_{I D X}}}\right),[12]
$$

364 where $\tau_{\sigma_{\xi}}$ and $\kappa_{\sigma_{\xi}}$ represent the mean and standard deviation of $\sigma_{\xi}$, respectively. We

365 fit all Bayesian models in this study using the R package 'rstan' (66) to interface with Stan (67)

366 in R (61). R package 'MCMCvis' (68) was used to summarize, visualize, and manipulate all

367 Bayesian model output. General data manipulation and processing was done using the

368 'tidyverse' family of R packages (69). For each model, we ran four chains for 8000 iterations

369 each with a warmup of 4000 iterations. For all models, Rhat $<=1.01$ and the number of effective

370 samples was $>400$ for all parameters. No models had divergent transitions (67). Weakly

371 informative priors were given for all parameters. Graphical posterior predictive checks were used

372 to check that data generated by the model were similar to the data used to fit the model (70).

373 Data simulated from the posterior predictive distribution were similar to the observed data (Fig.

374 S9).

For all model results in the main text, we present posterior mean estimates for parameters

376 as well as the $89 \%$ credible intervals, following $(71)$. The choice of $89 \%$ is arbitrary but serves to

377 quantify parameter uncertainty while avoiding any suggestion that Bayesian credible intervals

378 are analogous to tests of statistical significance (as might be assumed if using 95\% cutoffs). For 
379

380

381

382

each parameter, we also present the probability that a given parameter is positive (calculated as the proportion of the posterior that is greater than 0$)$ as $p(>0)$, or negative (the proportion of the posterior that is less than 0$)$ as $p(<0)$. Scenarios in which $p(>0)$ or $p(<0)$ are near 0.5 indicate that a positive relationship is equally likely as a negative relationship.

To create species maps for Fig. 2C, 3C, 4B, we used range maps obtained from (72). Estimated effects of latitude and elevation were used to predict values for Size Index and Wing Index across the range of these species. We excluded all areas greater than $2000 \mathrm{~m}$ of elevation (73) when deriving predictions for Size Index for red-eyed vireo (Vireo olivaceus) for Fig. 3C, to avoid making predictions outside the elevational range of this species in the Rocky Mountains.

\section{Body size as a function of temporal variation in temperature}

To quantify how intraspecific variation in size across time is influenced by temperature, we modeled $S I$ as a function $M T$ (June maximum temperature at each station). The response variable $\left(y_{T V T}\right)$ for capture $i$, banding station $j$, and species $k$ was modeled as $\mathrm{t}$ distributed, as a function of $M T$,

$$
\text { where } \alpha_{T V T} \text { is the species-station-specific intercept term, } \beta_{T V T} \text { is the species-station-specific }
$$
effect of temperature on the response variable, $\sigma_{T V T}$ is the species-specific process error, $v_{T V T}$ represents the degrees of freedom, and the TVT subscript denotes the association of that parameter with this model. Parameter $\alpha_{T V T}$ was modeled normally distributed, as a function of MST (deviations of June maximum temperature from species-specific range-wide temperature at each station), 


$$
\alpha_{T V T_{j k}} \sim N\left(\mu_{\alpha_{T V T} j k}, \sigma_{\alpha_{T V T}}\right)[14]
$$

403

$$
\mu_{\alpha_{T V T} k}=\rho_{T V T_{k}}+\zeta_{T V T_{k}} \times M S T_{j k}
$$

404

$$
\left[\begin{array}{c}
\rho_{T^{T T_{k}}} \\
\zeta_{T T_{k}}
\end{array}\right] \sim \operatorname{MVN}\left(\left[\begin{array}{l}
\mu_{\rho_{T V T}} \\
\mu_{\zeta_{T V T}}
\end{array}\right], \Sigma_{\alpha_{\mathrm{TVT}}}\right)
$$

405 where $\rho_{T V T}$ is the species-specific intercept term, $\zeta_{T V T}$ is the species-specific effects of MST on $406 \alpha_{T V T}$, and $\sigma_{\alpha_{T V T}}$ represents the process error. Parameters $\rho_{T V T}$ and $\zeta_{T V T}$ were modeled as

407 multivariate normal, with means $\mu_{\rho_{T V T}}$ and $\mu_{\zeta_{T V T}}$, respectively and covariance $\Sigma_{\alpha_{T V T}}$ (a $2 \times 2$

408 covariance matrix). Parameter $\beta_{T V T}$ was similarly modeled as a function of MST.

$$
\begin{gathered}
\beta_{T_{V T} T_{j k}} \sim N\left(\mu_{\beta_{T V T_{j k}}}, \sigma_{\beta_{T V T}}\right)[15] \\
\mu_{\beta_{T V T_{j k}}}=\gamma_{T V T_{k}}+\theta_{T V T_{k}} \times M S T_{j k} \\
{\left[\begin{array}{l}
\gamma_{T V T_{k}} \\
\theta_{T V T_{k}}
\end{array}\right] \sim \operatorname{MVN}\left(\left[\begin{array}{l}
\mu_{\gamma_{T V T}} \\
\mu_{\theta_{T V T}}
\end{array}\right], \Sigma_{\beta_{\mathrm{TVT}}}\right)}
\end{gathered}
$$

412 Both the intercept $\left(\alpha_{T V T}\right)$ and slope $\left(\beta_{T V T}\right)$ at each species-station were modeled as a function of

413 mean station temperature, because both the overall size and the effect of temporal variation in

414 temperature might be expected to vary across this gradient. Parameter $\sigma_{T V T}$ was modeled as half-

415 normal,

$$
\sigma_{T V T_{k}} \sim H N\left(\tau_{\sigma_{T V T}}, \kappa_{\sigma_{T V T}}\right),[16]
$$

417 where $\tau_{\sigma_{T V T}}$ and $\kappa_{\sigma_{T V T}}$ represent the mean and standard deviation of $\sigma_{T V T}$, respectively.

419 morphological data were collected (lag-0), as well as temperature one- (lag-1) and two-years

420 (lag-2) prior to data collection, to explore the effect of temperature on morphology (i.e., the

421 effect of temperature in year $\mathrm{t}, \mathrm{t}-1$, and $\mathrm{t}-2$ on morphology in year $\mathrm{t}$ ) during the potential

422 hatching summer and subsequent summers and to account for the uncertainty and variability in 
423 the ages of these birds (all of which were known to be adults). For each model, we ran four

424 chains for 6000 iterations each with a warmup of 3000 iterations.

426 Body size as a function of spatial variation in temperature

427 To quantify how intraspecific variation in size across space is influenced by temperature, we

428 modeled SI as a function of $M T$ (mean June maximum temperature at each station across all

429 years). The response variable $\left(y_{S V T}\right)$ for capture $i$, banding station $j$, and species $k$ was modeled

430 as t-distributed,

$$
y_{S V T_{i j k}} \sim t\left(v_{S V T}, \mu_{S V T_{i j k}}, \sigma_{S V T_{k}}\right)[17]
$$

$$
\mu_{S V T_{i j k}}=\alpha_{S V T_{k}}+\xi_{S V T}{ }_{j k}
$$

433 where $\alpha_{S V T}$ is the species-specific intercept term, $\xi_{S V T}$ is the species-station-specific intercept,

$434 \sigma_{S V T}$ is the species-specific processes error, $v_{S V T}$ represents the degrees of freedom, and $S V T$

435 denotes the association of each parameter with this model. Parameter $\alpha_{S V T}$ was modeled as

436 normally distributed,

$$
\alpha_{S V T_{k}} \sim N\left(\mu_{\alpha_{S V T}}, \sigma_{\alpha_{S V T}}\right),[18]
$$

438 where $\mu_{\alpha}$ and $\sigma_{\alpha}$ represent the mean and standard deviation of $\alpha_{S V T}$, respectively. Parameter

$439 \xi_{S V T}$ was modeled as normally distributed, as a function of $M T$,

$$
\xi_{S V T_{j k}} \sim N\left(\mu_{\xi_{S V T}, j}, \sigma_{\xi_{S V T}}\right)[19]
$$

$$
\mu_{\xi_{S V T_{j k}}}=\beta_{S V T_{k}} \times M T
$$

442 where $\beta_{S V T_{k}}$ is the species-specific effect of $M T$, and $\sigma_{\xi_{S V T}}$ is the process error. Parameter $\beta_{S V T}$

443 was modeled as normally distributed,

$$
\beta_{S V T_{k}} \sim N\left(\mu_{\beta_{S V T}}, \sigma_{\beta_{S V T}}\right),[20]
$$


445 where $\mu_{\beta_{S V T}}$ and $\sigma_{\beta_{S V T}}$ represent the mean and standard deviation of $\beta_{S V T}$, respectively. We ran

446 four chains for this model for 8000 iterations each with a warmup of 4000 iterations.

447 To assess how responses to temperature varied across species, we modeled the species-

448 specific effect of spatial variation of temperature on $S I\left(\widehat{\beta_{S V T}}\right.$; the posterior mean of $\beta_{S V T}[\mathrm{Eq}$.

449 19], derived from the above model) and associated uncertainty as a function of ST (mean cross-

450 station temperature within each species' range). Parameter $\widehat{\beta_{S V T}}$ was modeled as normally

451 distributed, with mean $\pi_{S R}$ and standard deviation $\sigma_{\widehat{\beta_{S V T}}}$ (the posterior standard deviation of $\beta_{S V T}$

452 [Eq. 19], derived from the above model),

$$
{\widehat{\beta_{S V T}}}_{k} \sim N\left(\pi_{\mathrm{SR}_{\mathrm{k}}}, \sigma_{\widehat{\beta S V T}_{k}}\right),[21]
$$

454 where SR denotes the association of each parameter with this model. In this way, the uncertainty

455 in the species-specific estimates of the spatial temperature effect is propagated through these

456 analyses. Parameter $\pi_{\mathrm{SVT}}$ was modeled as multivariate normal, as a linear function of $S T$, in a

457 manner that accounts for the phylogenetic non-independence between species [following ( 74 ,

458 75)],

459

460

461

$$
\begin{gathered}
\pi_{\mathrm{SR}_{\mathrm{k}}} \sim \operatorname{MVN}\left(\mu_{\pi_{\mathrm{SR}} k}, \Sigma_{\mathrm{SR}} \times \sigma_{\pi_{\mathrm{SR}}}\right)[22] \\
\mu_{\pi_{\mathrm{SR}}}=\gamma_{S R}+\theta_{S R} \times S T_{k} \\
\Sigma_{\mathrm{SR}}=\lambda_{S R} \times \Sigma_{d i s}+\left(1-\lambda_{S R}\right) \times I
\end{gathered}
$$

462 where $\gamma_{S R}$ is the intercept term, $\theta_{S R}$ is the effect of $S T$ on the response variable, and $\sigma_{\pi_{S R}}$ is the

463 process error. Parameter $\Sigma_{\text {dis }}$ is a phylogenetic covariance matrix, standardized such that the

464 diagonal elements have a value of 1 . The off-diagonal elements of $\Sigma_{\text {dis }}$ describe the pair-wise

465 phylogenetic distances between the 105 species included in this study. The phylogenetic

466 covariance matrix was calculated from a consensus phylogenetic tree [calculated using the 
467 'phytools' package (76) in R] based on 100 trees for the species of interest obtained from

468 BirdTree [(62) www.birdtree.org]. Parameter $\lambda_{S R}$ is Pagel's lambda (77), which represents the

469 degree to which phylogenetic relatedness contributes to variation in $\pi_{S R}$, where values near 0

470 (the lower bound of the parameter) indicate low phylogenetic signal and values near 1 (the upper

471 bound of the parameter) correspond to variation following a Brownian motion model of

472 evolution (75), and $I$ is an identity matrix. We ran this model for 1000 iterations with a warmup

473 of 500 iterations.

474

475 Back-transformation of effect sizes to trait space

476 Steps outlined by Eqs. 1-5 were implemented in reverse, to calculate the response of absolute

477 morphological measurements (body mass and wing length) to variation over time, latitude, and

478 elevation, using posterior estimates for the effects of these predictors on SI and WI. That is, for

479 each species the effect sizes (i.e., posterior estimates) of these covariates on SI and WI were

480 multiplied by the standard deviation of $x^{\prime}$ and $y^{\prime}\left(\sigma_{x \prime}\right.$ and $\sigma_{y^{\prime}}$, respectively),

$$
\phi_{x_{k}^{\prime}}=\phi_{S I_{k}} \times \sigma_{x_{k}^{\prime}}[23]
$$

$$
\phi_{y_{k}^{\prime}}=\phi_{W I_{k}} \times \sigma_{y_{k}^{\prime}}
$$

483 where $\phi_{S I}$ and $\phi_{W I}$ are the effect of a given covariate on $S I$ and $W I$, respectively, for each

484 species $(k)$, and $\phi_{x}$ and $\phi_{y}$, represent the unstandardized effects of the covariate for each

485 species. Parameters $\phi_{x}$ and $\phi_{y}$, were then rotated using the transpose of R (Eq. 3),

$$
\left[\begin{array}{l}
\phi_{L M_{k}} \\
\phi_{L W_{k}}
\end{array}\right]=R^{T}\left[\begin{array}{l}
\phi_{x^{\prime}}{ }_{k} \\
\phi_{{y^{\prime}}^{\prime}}
\end{array}\right],[24]
$$

487 where $\phi_{L M}$ and $\phi_{L W}$ represent the effect of a given covariate on the logged absolute

488 morphological metrics, $L M$ (logged mass) and $L W$ (logged wing length), for each species. This 
489

490

491

492

493

494

495

496

497

498

499

500

501

502

503

504

505

506

507

508

509

transformation has the effect of rotating data in the opposite direction of the rotation performed in Eq. 4. Since $\phi_{L M}$ and $\phi_{L W}$ represent an effect size in log space, when exponentiated, these metrics represent the multiplicative change in (unlogged) mass and wing length for each one-unit change in a given covariate. Subtracting one from this value and multiplying by 100 gives the percent change in that metric. To determine the percent change in mass $\left(\omega_{M}\right)$ and wing length $\left(\omega_{W}\right)$ over the temporal, latitudinal, and elevational range at which data were collected for each species, we exponentiated the product of $\phi_{L M}$ and $L$ (for mass) and the product of $\phi_{L W}$ and $L$ (for wing length), subtracted one, and multiplied by 100 ,

$$
\begin{gathered}
\omega_{M_{C O V_{k}}}=\left(\left(e^{\phi_{L M_{k}} \times L_{k}}\right)-1\right) \times 100[25] \\
\omega_{W_{\text {COV }_{k}}}=\left(\left(e^{\phi_{L W_{k}} \times L_{k}}\right)-1\right) \times 100
\end{gathered}
$$

where $L$ represents the total number of covariate units (i.e., 30 years, the latitudinal range in degrees for a given species, or the elevational range in meters for a given species), and $C O V$ represents time $\left(\omega_{M_{T I M E}}\right.$ or $\left.\omega_{W_{T I M E}}\right)$, latitude $\left(\omega_{M_{L A T}}\right.$ or $\left.\omega_{W_{L A T}}\right)$, or elevation $\left(\omega_{M_{E L E V}}\right.$ or $\left.\omega_{W_{E L E V}}\right)$. This was done at each iteration of the posterior for the estimated effect of year $\left(\beta_{I D X}\right.$; Eq. 6), latitude ( $\gamma_{I D X}$; Eq. 11), and elevation $\left(\theta_{I D X}\right.$; Eq. 11), providing a posterior distribution for $\omega_{M_{C O V}}$ and $\omega_{W_{C O V}}$. To calculate the cross-species mean percent change in mass and wing length, we calculated the mean of $\omega_{M_{C O V}}$ and $\omega_{W_{C O V}}$ across all species at each posterior iteration, represented by $\mu_{\omega_{M_{C O V}}}$ and $\mu_{\omega_{W_{C O V}}}$, respectively,

$$
\begin{gathered}
\mu_{\omega_{M_{C O V}}}=\frac{\sum_{k=1}^{N} \omega_{M_{C O V_{k}}}}{N}[26] \\
\mu_{\omega_{W_{C O V}}}=\frac{\sum_{k=1}^{N} \omega_{W_{C O V_{k}}}}{N}
\end{gathered}
$$

where $\mathrm{N}$ is the number of species. 
512 To compare the observed rates of phenotypic change in this study to observed rates of

513 evolutionary change in other taxa, we calculated change in logged mass in terms of haldanes $(h)$,

$$
h=\frac{\left(\frac{x_{2}}{s_{p}}\right)-\left(\frac{x_{1}}{s_{p}}\right)}{g},[27]
$$

515 where $x_{2}$ and $x_{1}$ are the mean values for a morphological trait of interest at two time points, $s_{p}$ is

516 the standard deviation of the traits (pooled across time), and $g$ is the number of generations that

517 are likely to have occurred between the two time points (78). This measure, first proposed by

518 (79), represents the magnitude of phenotypic change in standard deviations per generation.

$519 \quad$ For each species, we predicted logged mass at the beginning $\left(x_{1}\right)$ and end $\left(x_{2}\right)$ of the 30-

520 year study period by subtracting and adding $\phi_{L M_{T I M E}} \times 15$ (where $\phi_{L M_{T I M E}}$ is from Eq. 24,

521 representing change in logged mass per year), respectively, from mean logged mass. We

522 calculated the within-population standard deviation across all years at each station and took the

523 mean value of this standard deviation across stations $\left(s_{p}\right)$ for each species. We used information

524 on generation length from $(80)$ to calculate the number of generations (generation length / 30) for

525 a particular species over this time period $(g)$.

526 Prior work has suggested that rates of evolutionary change of $|h|=0.1-0.3$ standard

527 deviations per generation to be rapid (81), and that the maximal rate of phenotypic change that

528 can be sustained indefinitely is approximately 0.1 phenotypic standard deviations per generation

529 (82). For all species in this study, $|h|<0.1$. Rates of phenotypic change were similar to those

530 observed in other taxa undergoing anthropogenic disturbance (Fig. S10) (83). 


\section{REFERENCES}

533 1. D. Schluter, The evolution of finch communities on islands and continents: Kenya vs.

534 Galapagos. Ecol. Monogr. 58, 229-249 (1988).

535 2. J. L. Gardner, A. Peters, M. R. Kearney, L. Joseph, R. Heinsohn, Declining body size: a third universal response to warming? Trends Ecol. Evol. 26, 285-291 (2011).

3. C. Bergmann, Über die Verhältnisse der wärmeökonomie der Thiere zu ihrer grösse. Gött. Stud. 3, 595-708 (1847).

4. K. Riemer, R. P. Guralnick, E. P. White, No general relationship between mass and

5. D. M. Dehling, P. Jordano, H. M. Schaefer, K. Böhning-Gaese, M. Schleuning, Morphology predicts species' functional roles and their degree of specialization in plantfrugivore interactions. Proc. R. Soc. B Biol. Sci. 283, 20152444 (2016).

6. P. R. Grant, Inheritance of size and shape in a population of Darwin's finches, Geospiza

7. S. Des Roches, D. M. Post, N. E. Turley, J. K. Bailey, A. P. Hendry, M. T. Kinnison, J. A.

8. J. A. Allen, The influence of physical conditions in the genesis of species. Radic. Rev. 1, Schweitzer, E. P. Palkovacs, The ecological importance of intraspecific variation. Nat. Ecol. Evol. 2, 57-64 (2018).

9. D. L. Altshuler, R. Dudley, The physiology and biomechanics of avian flight at high altitude. Integr. Comp. Biol. 46, 62-71 (2006).

10. C. Teplitsky, V. Millien, Climate warming and Bergmann's rule through time: is there any evidence? Evol. Appl. 7, 156-168 (2014).

11. Y. Yom-Tov, S. Yom-Tov, J. Wright, C. J. R. Thorne, R. Du Feu, Recent changes in body weight and wing length among some British passerine birds. Oikos. 112, 91-101 (2006).

12. J. Van Buskirk, R. S. Mulvihill, R. C. Leberman, Declining body sizes in North American Winger, Shared morphological consequences of global warming in North American migratory birds. Ecol. Lett. 23, 316-325 (2020). Stanton, A. Panjabi, L. Helft, M. Parr, P. P. Marra, Decline of the North American avifauna. Science. 366, 120-124 (2019). 
15. D. F. DeSante, J. F. Saracco, D. R. O'Grady, K. M. Burton, B. L. Walker, Methodological considerations of the Monitoring Avian Productivity and Survivorship (MAPS) program. Stud. Avian Biol., 28-45 (2004).

16. G. B. West, J. H. Brown, B. J. Enquist, A general model for the origin of allometric scaling laws in biology. Science. 276, 122-126 (1997).

17. M. A. Ballinger, M. W. Nachman, The contribution of genetic and environmental effects to Bergmann's rule and Allen's rule in house mice. bioRxiv (2021).

18. S. C. Andrew, L. L. Hurley, M. M. Mariette, S. C. Griffith, Higher temperatures during development reduce body size in the zebra finch in the laboratory and in the wild. J. Evol. Biol. 30, 2156-2164 (2017).

19. A. M. Siepielski, M. B. Morrissey, S. M. Carlson, C. D. Francis, J. G. Kingsolver, K. D. Whitney, L. E. B. Kruuk, No evidence that warmer temperatures are associated with selection for smaller body sizes. Proc. R. Soc. B Biol. Sci. 286, 20191332 (2019).

20. E. A. Riddell, K. J. Iknayan, B. O. Wolf, B. Sinervo, S. R. Beissinger, Cooling requirements fueled the collapse of a desert bird community from climate change. Proc. Natl. Acad. Sci. 116, 21609-21615 (2019).

21. G. T. Pecl, M. B. Araújo, J. D. Bell, J. Blanchard, T. C. Bonebrake, I.-C. Chen, T. D. Clark, R. K. Colwell, F. Danielsen, B. Evengård, L. Falconi, S. Ferrier, S. Frusher, R. A. Garcia, R. B. Griffis, A. J. Hobday, C. Janion-Scheepers, M. A. Jarzyna, S. Jennings, J. Lenoir, H. I. Linnetved, V. Y. Martin, P. C. McCormack, J. McDonald, N. J. Mitchell, T. Mustonen, J. M. Pandolfi, N. Pettorelli, E. Popova, S. A. Robinson, B. R. Scheffers, J. D. Shaw, C. J. B. Sorte, J. M. Strugnell, J. M. Sunday, M.-N. Tuanmu, A. Vergés, C. Villanueva, T. Wernberg, E. Wapstra, S. E. Williams, Biodiversity redistribution under climate change: Impacts on ecosystems and human well-being. Science. 355, eaai9214 (2017).

22. D. J. Futuyma, Evolutionary constraint and ecological consequences. Evolution. 64, 18651884 (2010).

23. C. J. Murren, J. R. Auld, H. Callahan, C. K. Ghalambor, C. A. Handelsman, M. A. Heskel, J. G. Kingsolver, H. J. Maclean, J. Masel, H. Maughan, D. W. Pfennig, R. A. Relyea, S. Seiter, E. Snell-Rood, U. K. Steiner, C. D. Schlichting, Constraints on the evolution of phenotypic plasticity: limits and costs of phenotype and plasticity. Heredity. 115, 293-301 (2015).

24. M. W. Baldwin, H. Winkler, C. L. Organ, B. Helm, Wing pointedness associated with migratory distance in common-garden and comparative studies of stonechats (Saxicola torquata). J. Evol. Biol. 23, 1050-1063 (2010).

25. I. Newton, The migration ecology of birds (Elsevier, 2010). 
26. S. M. Clegg, J. F. Kelly, M. Kimura, T. B. Smith, Combining genetic markers and stable isotopes to reveal population connectivity and migration patterns in a Neotropical migrant, Wilson's warbler (Wilsonia pusilla). Mol. Ecol. 12, 819-830 (2003).

27. C. P. Bell, Leap-frog migration in the fox sparrow: Minimizing the cost of spring migration. The Condor. 99, 470-477 (1997).

28. S. Billerman, B. Keeney, P. Rodewald, T. Schulenberg, Birds of the World. Ithaca N. Y. Cornell Lab. Ornithol. (2020).

29. A. Desrochers, Morphological response of songbirds to 100 years of landscape change in North America. Ecology. 91, 1577-1582 (2010).

30. J. P. Swaddle, R. Lockwood, Morphological adaptations to predation risk in passerines. $J$. Avian Biol. 29, 172-176 (1998).

31. S. L. Chown, C. J. Klok, Altitudinal body size clines: latitudinal effects associated with changing seasonality. Ecography. 26, 445-455 (2003).

32. W. A. Boyle, Altitudinal bird migration in North America. The Auk. 134, 443-465 (2017).

33. A. R. Spence, M. W. Tingley, Body size and environment influence both intraspecific and interspecific variation in daily torpor use across hummingbirds. Funct. Ecol. 35, 870-883 (2021).

34. R. E. Moreau, Variation in the western Zosteropidae (Aves). Bull. Br. Mus. Nat. Hist. Zool. 4, 311-433 (1957).

35. T. H. Hamilton, The adaptive significances of intraspecific trends of variation in wing length and body size among bird species. Evolution. 15, 180-194 (1961).

36. I. D. Hodkinson, Terrestrial insects along elevation gradients: species and community responses to altitude. Biol. Rev. 80, 489-513 (2005).

37. P. Feinsinger, R. K. Colwell, J. Terborgh, S. B. Chaplin, Elevation and the morphology, flight energetics, and foraging ecology of tropical hummingbirds. Am. Nat. 113, 481-497 (1979).

38. J. W. Aldrich, Ecogeographical variation in size and proportions of song sparrows (Melospiza melodia). Ornithol. Monogr., 1-134 (1984).

39. Y. Sun, M. Li, G. Song, F. Lei, D. Li, Y. Wu, The role of climate factors in geographic variation in body mass and wing length in a passerine bird. Avian Res. 8, 1-9 (2017).

40. C. Sheard, M. H. C. Neate-Clegg, N. Alioravainen, S. E. I. Jones, C. Vincent, H. E. A. MacGregor, T. P. Bregman, S. Claramunt, J. A. Tobias, Ecological drivers of global gradients in avian dispersal inferred from wing morphology. Nat. Commun. 11 (2020). 
41. S. Des Roches, L. H. Pendleton, B. Shapiro, E. P. Palkovacs, Conserving intraspecific variation for nature's contributions to people. Nat. Ecol. Evol. 5, 574-582 (2021).

42. E. A. Riddell, K. J. Iknayan, B. O. Wolf, B. Sinervo, S. R. Beissinger, Cooling requirements fueled the collapse of a desert bird community from climate change. Proc. Natl. Acad. Sci. 116, 21609-21615 (2019).

43. A. E. McKechnie, B. O. Wolf, Climate change increases the likelihood of catastrophic avian mortality events during extreme heat waves. Biol. Lett. 6, 253-256 (2010).

44. S. R. Conradie, S. M. Woodborne, S. J. Cunningham, A. E. McKechnie, Chronic, sublethal effects of high temperatures will cause severe declines in southern African arid-zone birds during the 21 st century. Proc. Natl. Acad. Sci. 116, 14065-14070 (2019).

45. V. Radchuk, T. Reed, C. Teplitsky, M. van de Pol, A. Charmantier, C. Hassall, P. Adamík, F. Adriaensen, M. P. Ahola, P. Arcese, J. Miguel Avilés, J. Balbontin, K. S. Berg, A. Borras, S. Burthe, J. Clobert, N. Dehnhard, F. de Lope, A. A. Dhondt, N. J. Dingemanse, H. Doi, T. Eeva, J. Fickel, I. Filella, F. Fossøy, A. E. Goodenough, S. J. G. Hall, B. Hansson, M. Harris, D. Hasselquist, T. Hickler, J. Joshi, H. Kharouba, J. G. Martínez, J.-B. Mihoub, J. A. Mills, M. Molina-Morales, A. Moksnes, A. Ozgul, D. Parejo, P. Pilard, M. Poisbleau, F. Rousset, M.-O. Rödel, D. Scott, J. C. Senar, C. Stefanescu, B. G. Stokke, T. Kusano, M. Tarka, C. E. Tarwater, K. Thonicke, J. Thorley, A. Wilting, P. Tryjanowski, J. Merilä, B. C. Sheldon, A. Pape Møller, E. Matthysen, F. Janzen, F. S. Dobson, M. E. Visser, S. R. Beissinger, A. Courtiol, S. Kramer-Schadt, Adaptive responses of animals to climate change are most likely insufficient. Nat. Commun. 10 (2019).

46. E. A. Riddell, K. J. Iknayan, L. Hargrove, S. Tremor, J. L. Patton, R. Ramirez, B. O. Wolf, S. R. Beissinger, Exposure to climate change drives stability or collapse of desert mammal and bird communities. Science. 371, 633-636 (2021).

47. M. W. Tingley, W. B. Monahan, S. R. Beissinger, C. Moritz, Birds track their Grinnellian niche through a century of climate change. Proc. Natl. Acad. Sci. 106, 19637-19643 (2009).

48. C. Youngflesh, J. Socolar, B. R. Amaral, A. Arab, R. P. Guralnick, A. H. Hurlbert, R. LaFrance, S. J. Mayor, D. A. W. Miller, M. W. Tingley, Migratory strategy drives specieslevel variation in bird sensitivity to vegetation green-up. Nat. Ecol. Evol. 5, 987-994 (2021).

49. L. Blueweiss, H. Fox, V. Kudzma, D. Nakashima, R. Peters, S. Sams, Relationships between body size and some life history parameters. Oecologia. 37, 257-272 (1978).

50. M. Kleiber, Body size and metabolic rate. Physiol. Rev. 27, 511-541 (1947).

51. P. Yodzis, S. Innes, Body size and consumer-resource dynamics. Am. Nat. 139, 1151-1175 (1992). 
52. R. O. Prum, Interspecific social dominance mimicry in birds: Social mimicry in birds. Zool. J. Linn. Soc. 172, 910-941 (2014).

53. P. Pyle, Identification guide to North American birds: a compendium of information on identifying, ageing, and sexing" near-passerines" and passerines in the hand (Slate Creek Press, 1997).

54. C. Leys, C. Ley, O. Klein, P. Bernard, L. Licata, Detecting outliers: Do not use standard deviation around the mean, use absolute deviation around the median. J. Exp. Soc. Psychol. 49, 764-766 (2013).

55. J. J. Danielson, D. B. Gesch, Global multi-resolution terrain elevation data 2010 (GMTED2010) (US Department of the Interior, US Geological Survey, 2011).

56. M. M. Thornton, R. Shrestha, Y. Wei, P. E. Thornton, S. Kao, B. E. Wilson, Daymet: Daily surface weather data on a 1-km grid for North America, version 4 (ORNL Distributed Active Archive Center, 2020).

57. C. H. Greenewalt, The flight of birds: the significant dimensions, their departure from the requirements for dimensional similarity, and the effect on flight aerodynamics of that departure. Trans. Am. Philos. Soc. 65, 1-67 (1975).

58. G. Longo, M. Montévil, Perspectives on Organisms: Biological Time, Symmetries, and Singularities (Springer, 2014).

59. P. H. Harvey, "Why and how phylogenetic relationships should be incorporated into studies of scaling" in Scaling in biology (Oxford University Press, 2000), p. 253-265.

60. D. Orme, R. Freckleton, G. Thomas, T. Petzoldt, S. Fritz, N. Isaac, W. Pearse, The caper package: comparative analysis of phylogenetics and evolution in R. R Package Version. 5, $1-36$ (2013).

61. R Core Team, R: A language and environment for statistical computing (R Foundation for Statistical Computing, Vienna, Austria, 2021; https://www.R-project.org/).

62. W. Jetz, G. H. Thomas, J. B. Joy, K. Hartmann, A. O. Mooers, The global diversity of birds in space and time. Nature. 491, 444-448 (2012).

63. R. L. Nudds, G. W. Kaiser, G. J. Dyke, Scaling of avian primary feather length. PLoS ONE. 6, e15665 (2011).

64. R. Nudds, Wing-bone length allometry in birds. J. Avian Biol. 38, 515-519 (2007).

65. S. C. Anderson, T. A. Branch, A. B. Cooper, N. K. Dulvy, Black-swan events in animal populations. Proc. Natl. Acad. Sci. 114, 3252-3257 (2017).

66. Stan Development Team. 2018. Stan Modeling Language Users Guide and Reference Manual, Version 2.18.0. http://mc-stan.org. 
67. B. Carpenter, A. Gelman, M. D. Hoffman, D. Lee, B. Goodrich, M. Betancourt, M. Brubaker, J. Guo, P. Li, A. Riddell, Stan: A probabilistic programming language. J. Stat. Softw. 76 (2017).

68. C. Youngflesh, MCMCvis: Tools to visualize, manipulate, and summarize MCMC output. J. Open Source Softw. 3, 640 (2018).

69. H. Wickham, M. Averick, J. Bryan, W. Chang, L. McGowan, R. François, G. Grolemund, A. Hayes, L. Henry, J. Hester, M. Kuhn, T. Pedersen, E. Miller, S. Bache, K. Müller, J. Ooms, D. Robinson, D. Seidel, V. Spinu, K. Takahashi, D. Vaughan, C. Wilke, K. Woo, H. Yutani, Welcome to the Tidyverse. J. Open Source Softw. 4, 1686 (2019).

70. J. Gabry, D. Simpson, A. Vehtari, M. Betancourt, A. Gelman, Visualization in Bayesian workflow. J. R. Stat. Soc. Ser. A Stat. Soc. 182, 389-402 (2019).

71. R. McElreath, Statistical rethinking: A Bayesian course with examples in R and Stan (Chapman and Hall/CRC, 2018).

72. BirdLife International. 2019. Data zone. Available from http://www.birdlife.org/datazone/index.html.

73. S. Cramp, C. Perrins, Handbook of the birds of Europe, the Middle East and North Africa. The birds of the Western Palearctic. Crows to finches, vol. VIII. (Oxford University Press, 1994).

74. J. Che-Castaldo, C. Che-Castaldo, M. C. Neel, Predictability of demographic rates based on phylogeny and biological similarity. Conserv. Biol. 32, 1290-1300 (2018).

75. P. de Villemereuil, J. A. Wells, R. D. Edwards, S. P. Blomberg, Bayesian models for comparative analysis integrating phylogenetic uncertainty. BMC Evol. Biol. 12, 102 (2012).

76. L. J. Revell, phytools: an R package for phylogenetic comparative biology (and other things): phytools: R package. Methods Ecol. Evol. 3, 217-223 (2012).

77. M. Pagel, Inferring the historical patterns of biological evolution. Nature. 401, 877-884 (1999).

78. A. P. Hendry, M. T. Kinnison, Perspective: The pace of modern life: measuring rates of contemporary microevolution. Evolution. 53, 1637-1653 (1999).

79. P. Gingerich, Rates of evolution: effects of time and temporal scaling. Science. 222, 159$162(1983)$.

80. J. P. Bird, R. Martin, H. R. Akçakaya, J. Gilroy, I. J. Burfield, S. Garnett, A. Symes, J. Taylor, Ç. H. Şekercioğlu, S. H. M. Butchart, Generation lengths of the world's birds and their implications for extinction risk. Conserv. Biol. (2020).

81. P. D. Gingerich, Rates of evolution. Annu. Rev. Ecol. Evol. Syst. 40, 657-675 (2009). 
82. R. Bürger, M. Lynch, Evolution and extinction in a changing environment: A quantitativegenetic analysis. Evolution. 49, 151-163 (1995).

83. A. P. Hendry, T. J. Farrugia, M. T. Kinnison, Human influences on rates of phenotypic change in wild animal populations. Mol. Ecol. 17, 20-29 (2008).

743 Acknowledgments: We thank MAPS station operators for collecting and sharing their data.

744 Dani Kaschube provided critical data access, assisted by Rob Guralnick and Rafael LaFrance.

745 We thank Christian Che-Castaldo for helpful discussions regarding the statistical modeling.

746 Illustrations were provided by Lauren Helton.

747 Funding: National Science Foundation grant EF 1703048 (MWT)

748 National Science Foundation grant EF 2033263 (MWT)

749 Author contributions: CY led formal analysis, and CY and MWT shared conceptualization and

750 writing of the original draft. RBS and JFS facilitated data access. All authors contributed to

751 review and editing of drafts.

752 Competing interests: The authors declare no competing interests.

753 Data and Materials availability: All code used to produce analyses are freely available on

754 Github (https://github.com/caseyyoungflesh/MAPS_morph_changes) and will be archived on

755 Zenodo upon acceptance. Data from the Monitoring Avian Productivity and Survivorship

756 (MAPS) program are curated and managed by The Institute for Bird Populations and were

757 queried from the MAPS database on 2019-10-16. MAPS data will be archived on Dryad upon

758 acceptance.

\section{Supplementary Materials:}

760 Figs. S1 to S10

761 Tables S1 to S4 

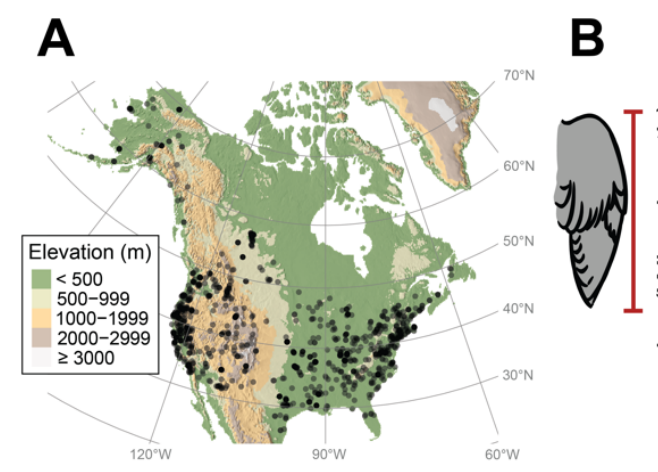

763

Fig. 1. The Monitoring Avian Productivity and Survivorship (MAPS) dataset provides an

\section{unparalleled resource for studying avian morphological variation over time and space. (A)}

765

766

767

768

769

770

771

772

773

774

775

$776 \quad$ Fig. S2.

Data on individual birds come from 1124 MAPS banding stations (black points) spanning the latitudinal and elevational extent of North America. (B) Measurements were taken for both wing length (chord of the unflattened wing) and mass for each captured bird. Based on allometric scaling principals and empirical measurements across species, wing length is expected to be proportional to mass to the $1 / 3$ power (the scaling exponent in the power law equation); logging both variables linearizes this relationship. Points represent individuals from a single hypothetical species. (C) The scaling exponent was used to create a rotation matrix which was applied to logged wing length and logged mass for each species, to derive two independent morphological indices: a Size Index $(S I)$ and a Wing Index $(W I)$, denoting the overall size of each individual bird and the degree to which wing length deviates from its expected value given the body mass of the individual, respectively. For additional details on this mathematical transformation, see Fig. S2.

777 


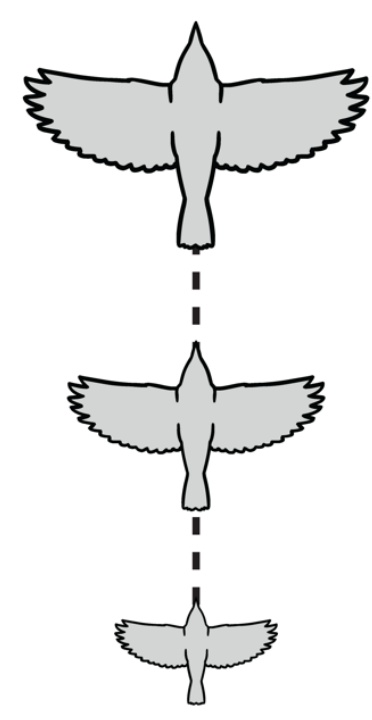

779

780

781

782

783

784

785

786

787

788

789 maximum temperature in a single year, 2018.
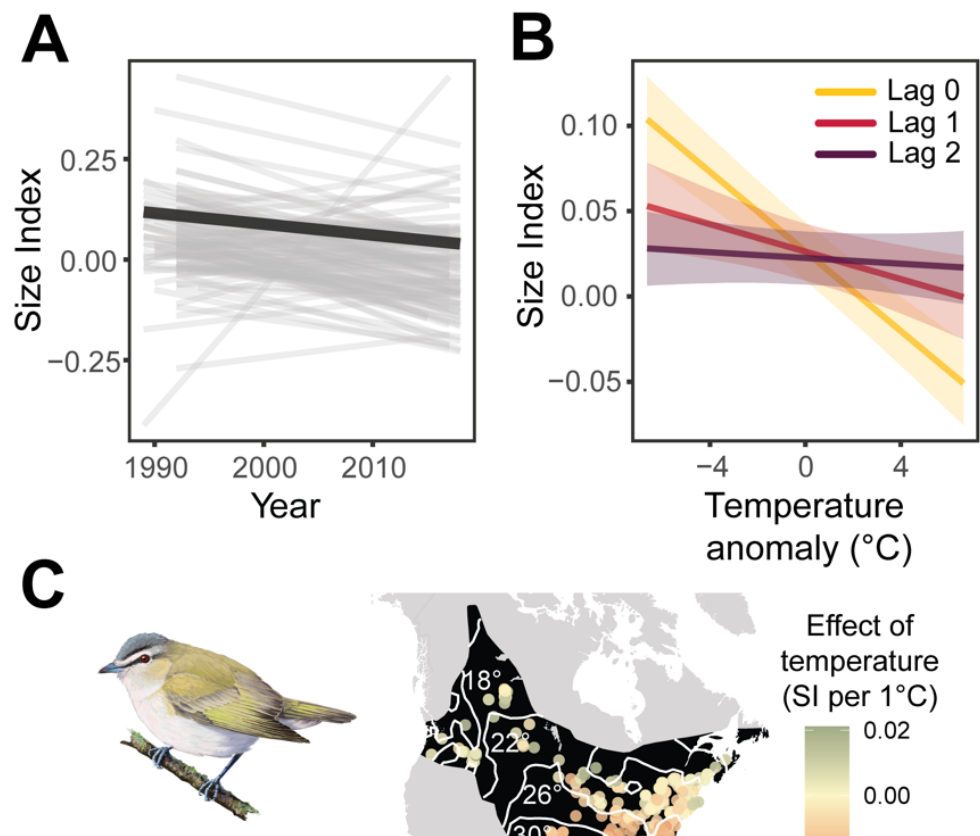

Red-eyed vireo Vireo olivaceus

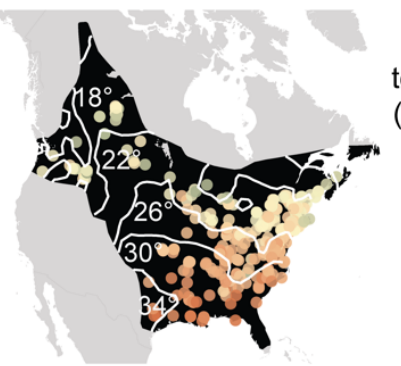

Effect of temperature $\left(\mathrm{SI}\right.$ per $\left.1^{\circ} \mathrm{C}\right)$

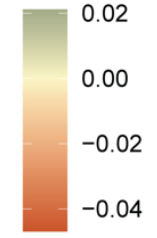

Fig. 2: Change in Size Index $(S I)$ over time and in response to temporal fluctuations in temperature. (A) Change in SI over time for 105 species, controlling for changes over latitude and elevation. Each thin gray line represents the trend for one species, while the thick black line represents the mean trend across all species. (B) Change in $S I$ across species in response to interannual fluctuations in June maximum temperature in the year of capture (Lag 0) as well as one (Lag 1) and two years (Lag 2) prior to capture. Ribbons represent 89\% CIs. (C) Effect of $1{ }^{\circ} \mathrm{C}$ change in temperature on SI at capture locations for a representative species, the red-eyed vireo Vireo olivaceus, showing stronger effects of temperature on SI in warmer areas. Darker, orange hues represent a stronger negative effect of temperature on SI. The black polygon represents the range of the species, while white lines (and associated white text) represent isoclines for June 


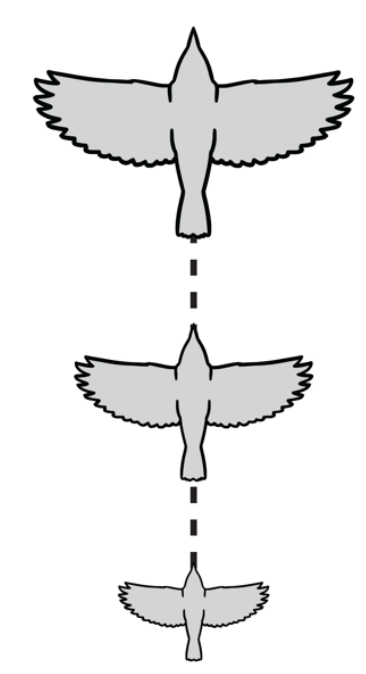

A
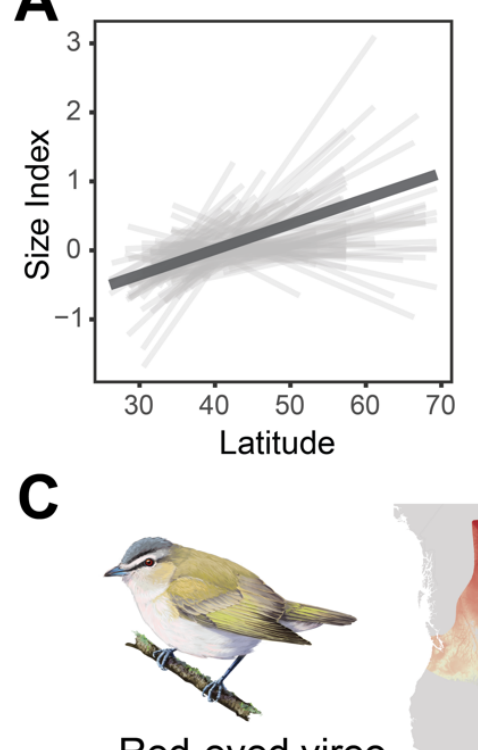

B

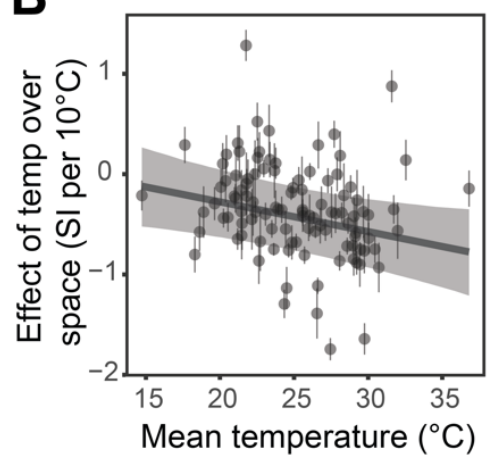

Red-eyed vireo

Vireo olivaceus
Predicted

Size Index

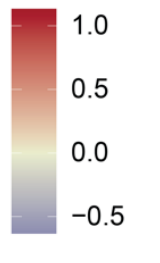

792 Fig. 3: Change in SI over latitude and in response to spatial variation in temperature. (A)

793 Change in SI over latitude for 105 species, controlling for changes over time and elevation. Each

794 thin gray line represents the trend for one species, while the thick black line represents the mean

795 trend across all species. (B) The effect of spatial variation in temperature on SI within each

796 species as a function of the mean (range-wide) temperature experienced by that species. Each

797 point represents a single species. Gray vertical bars represent one posterior standard deviation of

798 the effect of spatial variation in temperature on $S I$, the thick black line represents the linear

799 model fit, and the gray ribbon represents the $89 \%$ CI. (C) Predicted body size $(S I)$ over the range

800 of a representative species, the red-eyed vireo Vireo olivaceus, based on the estimated effect of

801 latitude and elevation. Yellow hues represent average, red hues represent larger than average,

802 and blue hues represent smaller than average predicted $S I$. 

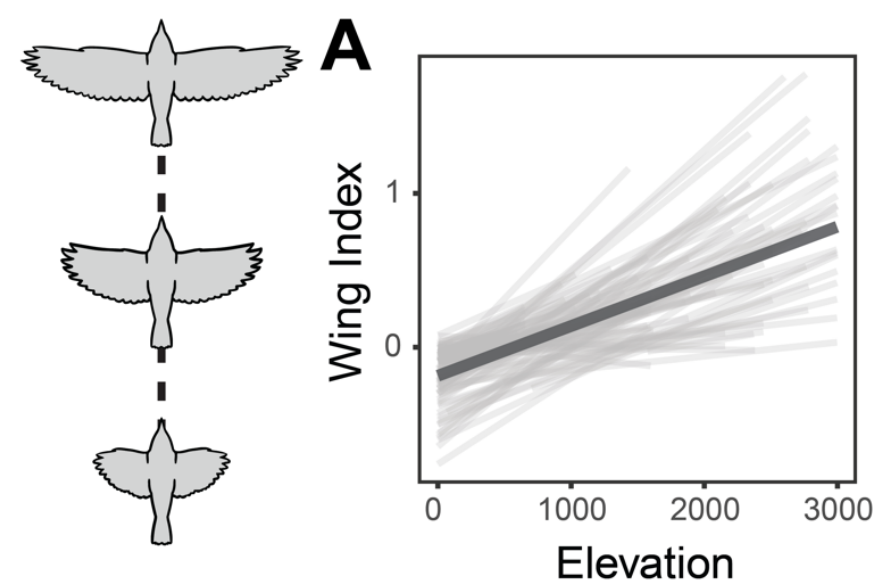

B Northern parula

Setophaga americana

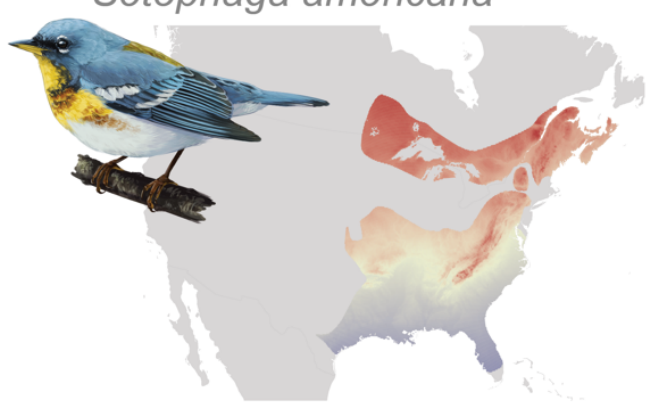

Predicted Wing Index

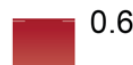

0.4

0.2

0.0

$-0.2$

805 Fig. 4: Change in WI over elevation and across latitude. (A) Change in WI over elevation for

806105 species, controlling for changes over time and latitude. Each thin gray line represents the

807 trend for one species, while the thick black line represents the mean trend across all species. (B)

808 Predicted wingyness $(W I)$ over the range of a representative species, the northern parula

809 Setophaga americana, based on the estimated effect of latitude and elevation. Yellow hues

810 represent average, red hues represent larger than average, and blue hues represent smaller than

811 average predicted $W I$.

812 